TITLE:

\title{
Three-Dimensional Simulation of SOFC Anode Polarization Characteristics Based on Sub-Grid Scale Model
}

\section{AUTHOR(S):}

Kishimoto, Masashi; Iwai, Hiroshi; Saito, Motohiro; Yoshida, Hideo

\section{CITATION:}

Kishimoto, Masashi ... [et al]. Three-Dimensional Simulation of SOFC Anode Polarization Characteristics Based on Sub-Grid Scale Model. ECS Transactions 2011, 35(1): 831-840

\section{ISSUE DATE:}

2011

URL:

http://hdl.handle.net/2433/163421

RIGHT:

(c) 2011 ECS - The Electrochemical Society 


\section{Three-Dimensional Simulation of SOFC Anode Polarization Characteristics Based on Sub-Grid Scale Model}

Masashi Kishimoto, Hiroshi Iwai, Motohiro Saito and Hideo Yoshida

ECS Trans. 2011, Volume 35, Issue 1, Pages 831-840.

doi: $10.1149 / 1.3570064$
Email alerting
Receive free email alerts when new articles cite this article - sign up in the service box at the top right corner of the article or click here




\title{
Three-Dimensional Simulation of SOFC Anode Polarization Characteristics Based on Sub-Grid Scale Model
}

\author{
Masashi Kishimoto, Hiroshi Iwai, Motohiro Saito, Hideo Yoshida \\ Department of Aeronautics and Astronautics, Kyoto University, Kyoto 606-8501, Japan
}

\begin{abstract}
Three-dimensional numerical simulation of SOFC anode polarization is conducted with a structure obtained by a focused ion beam and scanning electron microscope (FIB-SEM). Electronic, ionic and gaseous transports with electrochemical reaction are considered. A sub-grid scale (SGS) model is newly developed and effectively used to evaluate the transport flux in the porous structure. The proposed SGS model shows its potential to reasonably evaluate the transport flux considering the microstructure smaller than the grid size.
\end{abstract}

\section{Introduction}

The electrode microstructure of an SOFC has a significant influence on its power generation performance. Therefore, it is important to find the quantitative relationships between the electrode microstructure and performance to improve SOFCs. A focused ion beam and scanning electron microscope (FIB-SEM) technique are powerful means to directly observe the 3D microstructure of SOFC electrodes (1-4). From the obtained 3D structure, we can precisely evaluate many microstructural parameters which directly affect electrode performance.

As an application of the valuable data obtained by the FIB-SEM, development of a reliable and effective simulation model to predict the electrode performance is strongly required to clarify the effects of microstructure on performance. We (5) recently conducted a 1D numerical simulation and predicted the anode polarization characteristics; microstructural parameters obtained from a FIB-SEM dataset were applied in the simulation. In the 1D approach, however, some of the structural information, such as structural non-uniformity, is inevitably neglected. On the other hand, Shikazono et al. (6) applied lattice Boltzmann method (LBM) to predict the anode polarization characteristics using 3D microstructure obtained by the FIB-SEM. Also, Shearing et al. (7) applied volume of fluid (VOF) method for the similar purpose. Although these 3D simulations provide detailed information of the complicated phenomena in the electrodes, it is difficult to directly apply the image voxel of the FIB-SEM observation (length scale $50 \mathrm{~nm}$ ) as a grid system of the simulation because of extremely high calculation cost. To reduce the computational load, it is a common practice today to resample the original FIB-SEM dataset to make a calculation dataset with a lower spatial resolution. The resampling process raises a question whether the resampled dataset is still a good representation of the original microstructure because the structure smaller than the calculation grid size may be lost in the process.

In this study, we newly introduce a sub-grid scale (SGS) model, in which the grid size is relatively larger than that of the image voxel; simultaneously, the information of the 
microstructures, whose characteristic scale is smaller than the grid size, are taken into account within each grid. Specifically, in the SGS model, not only the volume conservation of each porous component is locally satisfied, but also higher-order quantities associated with the structural inhomogeneity is evaluated. We investigate the amount of transport flux through the porous anode with, or without the SGS model, and validate the applicability of the models.

\section{FIB-SEM Imaging}

In this study, we examine the conventional Ni-YSZ cermet anode (Ni:YSZ=50:50vol.\%, YSZ: $8 \mathrm{~mol}^{2} \mathrm{Y}_{2} \mathrm{O}_{3}-\mathrm{ZrO}_{2}$ ) of a button cell. The anode material, $\mathrm{NiO}-\mathrm{YSZ}$, is screen-printed on a YSZ disk electrolyte (thickness: $500 \mu \mathrm{m}$, TOSOH Co.) and sintered at $1673 \mathrm{~K}$ for $5 \mathrm{~h}$. After the anode is reduced at $1273 \mathrm{~K}$ with pure hydrogen, it is infiltrated with epoxy resin (Marumoto Struers KK) under vacuum conditions so that the pores of the porous electrode can be easily distinguished during SEM observation. Cured sample is polished using an Ar-ion beam cross-section polisher (JEOL Ltd., SM-09010) and provided to the FIB-SEM observation.

The 3D microstructure of the Ni-YSZ anode is observed by the FIB-SEM system. The FIB-SEM system, NVision 40, is equipped with a Gemini FE-SEM column (Zeiss), a zeta FIB column (SIINT) and a multichannel gas injection system (SIINT). An in-lens secondary electron detector is used for the microstructural observation with an acceleration voltage around 1 to $2 \mathrm{kV}$. Figure 1(a) shows an example of the obtained cross-sectional images. In the images, white, gray and black parts correspond to Ni, YSZ and pore phases, respectively.

The set of cross-sectional images obtained by the FIB-SEM tends to include misalignment between the images. Therefore, we put lines on the sample surface before the observation and use them for an alignment mark. We extract regions available for the later analysis and conduct the phase separation on the basis of the brightness of the images. After the alignment and the phase separation, the sequential set of $2 \mathrm{D}$ images is lined up with the actual increment and the 3D porous microstructure is reconstructed in a virtual field. Figure 1(b) shows the reconstructed microstructure of the porous anode. We use the commercial image processing software, Avizo (Mercury Computer Systems, Inc.), for the phase separation, 3D reconstruction, and some of the quantification explained afterward.

(a)

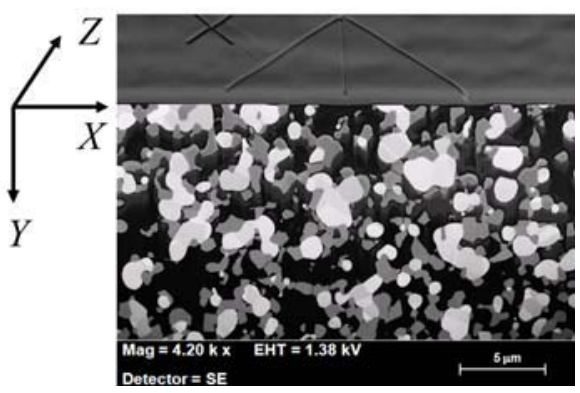

(b)

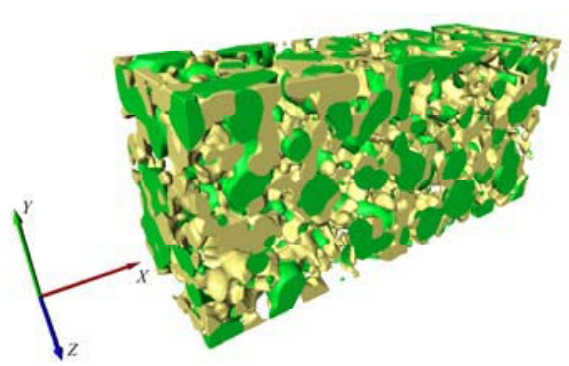

Figure 1. FIB-SEM observation of the SOFC porous anode. (a) Example of the cross-sectional images. (b) Reconstructed 3D structure (Green:Ni, Yellow:YSZ). 


\section{Sub-Grid Scale Model}

We conduct a numerical simulation of anode polarization characteristics with $3 \mathrm{D}$ porous structure obtained by the FIB-SEM. The simulation is based on finite volume method (FVM). We consider the conservation of electrons in Ni phase, oxygen ions in YSZ phase and gas components in pore phase and also electrochemical oxidation of hydrogen at TPBs. Temperature is assumed to be constant and uniform over the whole anode region.

We divide the anode region into a number of control volumes and use them as calculation grids. Microstructural parameters, such as volume fractions, particle and pore diameters and TPB density are quantified inside each control volume (Fig. 2). In this process, most of the structural information whose characteristic scale is smaller than grid size is lost. Therefore, as far as the meshing procedure is involved, structural information is inevitably changed, which may reduce the accuracy of the simulation. For this problem, we propose to use a model, which keeps the sub-gird-scale information of porous structure in the calculation domain. In this paper, we introduce the following two different SGS models and compare the results.

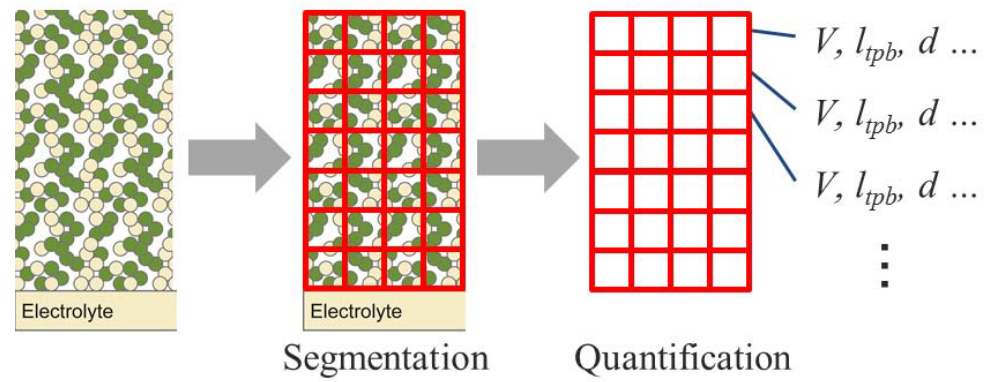

Figure 2. Schematic picture of grid generation.

\section{SGS Model 1: Volume Conservation}

As the simplest SGS model, we consider the conservation of the phase volume in each control volume. The numbers of voxels corresponding to Ni, YSZ and pore phases are counted and volume fractions are obtained in each grid. By using the volume fraction, effective transport coefficient, $\Gamma_{i}^{e f f}$, are defined as the following formula:

$$
\Gamma_{i}^{e f f}=V_{i} \Gamma_{i}
$$

where $\Gamma_{i}$ is the bulk transport coefficient and $V_{i}$ is the volume fraction of phase $i$.

Also, TPB density and pore diameter are evaluated in each control volume. TPB is detected as the edge line among Ni, YSZ and pore voxels, and the sum of the length is measured in each grid. This method, however, overestimates the total TPB length, because the actual TPB has much smoother shape. Therefore, we applied the scaling correction so that the total TPB length is the same as that obtained from the whole sample volume by volume expansion method (3). Pore diameter is evaluated by the line intercept method $(8,9)$. Lines are drawn in three directions along the orthogonal coordinate, whose origin is the focused voxel. The length of the lines intercepted by pore phase is measured 
and averaged to be used as the average pore diameter. We refer this model as SGS1 in the later sections.

\section{SGS Model 2: Phase Connectivity}

In addition to those considered in the SGS1, flux correction is performed in the SGS model 2 (SGS2). The correction is based on cross-sectional area of conductive volume on grid interface. In the SGS1, transport flux is falsely induced even if there is no conductive volume on the shared grid interface of the neighboring grids. Such an unrealistic flux may cause overestimation of transport, and deteriorate accuracy of the simulation. In the SGS2, the amount of flux between neighboring grids is evaluated in proportion to the cross-sectional area of conductive volume existing on the grid interface. The flux correction is schematically illustrated in Fig. 3. Effective transport coefficient is obtained as the following formula:

$$
\Gamma_{i}^{e f f}=\frac{S_{i}}{S_{\text {all }}} V_{i} \Gamma_{i}
$$

where $S$ is the surface area on the grid interface.

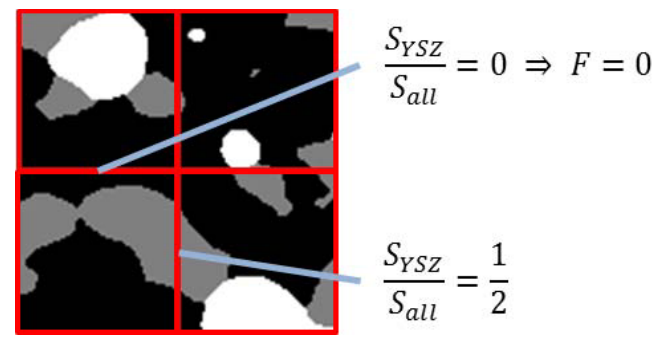

Figure 3. Flux correction based on cross-sectional area on grid interface.

\section{Governing Equations}

\section{$\underline{\text { Electron and Ion Transport }}$}

In the SOFC anodes, electrons and oxygen ions are transported through the Ni phase and YSZ phase, respectively. Considering the conservation of these species, the following equations are introduced as governing equations:

$$
\begin{aligned}
& \nabla \cdot\left(\sigma_{e l}^{e f f} \nabla \phi_{e l}\right)=-i_{t p b} \\
& \nabla \cdot\left(\sigma_{i o}^{e f f} \nabla \phi_{i o}\right)=i_{t p b}
\end{aligned}
$$

where $\phi_{e l}$ and $\phi_{i o}$ are the electric potential in electron-conductive phase (Ni) and oxygen-ion-conductive phase (YSZ), respectively. $i_{t p b}$ is the current exchanged between the two phases. $\sigma_{e l}^{e f f}$ and $\sigma_{i o}$ eff are effective electron/ion conductivities, which are evaluated depending on the applied SGS models (eqs.[1, 2]). Bulk conductivities are found in literature $(10,11)$. 


\section{Diffusion of Gas Species}

Gaseous diffusion of hydrogen and steam is considered on the basis of a dusty-gas model (DGM) $(12,13)$. If a constant total pressure is assumed, the DGM is expressed as follows:

$$
\frac{N_{i}}{D_{i, \mathrm{~K}}^{e f f}}+\sum_{j \neq i} \frac{X_{j} N_{i}-X_{i} N_{j}}{D_{i j}^{e f f}}=\frac{P_{t}}{R T} \nabla X_{i}
$$

where $X_{i}, N_{i}$ and $P_{i}$ are molar fraction, molar flux and partial pressure of species $i$, respectively. $P_{t}$ is the total pressure. $D_{i, \mathrm{~K}}^{\text {eff }}$ and $D_{i j}{ }^{e f f}$ are the effective Knudsen diffusion coefficient and the effective binary diffusion coefficient which are evaluated depending on the applied SGS models (eqs.[1, 2]). For the bulk binary diffusion coefficient, the Fuller-Schettler-Giddings' equation (14) is adopted in this study:

$$
D_{i j}=\frac{0.01013 T^{1.75}\left(\frac{1}{M_{i} \times 10^{3}}+\frac{1}{M_{j} \times 10^{3}}\right)^{1 / 2}}{P_{t}\left[\left(\Sigma v_{i} \times 10^{6}\right)^{1 / 3}+\left(\Sigma v_{j} \times 10^{6}\right)^{1 / 3}\right]^{2}}
$$

where $M_{i}$ is the molecular mass, and $\Sigma v_{i}$ represents the diffusion volume of the molecules of species $i(15)$. The bulk Knudsen diffusion coefficient is estimated by using the local pore diameter $d_{p}$ as follows:

$$
D_{i, \mathrm{~K}}=\frac{d_{p}}{2} \frac{2}{3} \sqrt{\frac{8 R T}{\pi M_{i}}}
$$

\section{Electrochemical Reaction}

In SOFC anodes, electrochemical oxidation of hydrogen takes place at the TPBs. Local charge-transfer rate for the hydrogen oxidation within the anode is represented by Butler-Volmer equation as follows:

$$
i_{t p b}=i_{0}\left[\exp \left(\frac{F \eta_{a c t}}{R T}\right)-\exp \left(-\frac{F \eta_{a c t}}{R T}\right)\right]
$$

where $i_{0}$ is the exchange current density, and $\eta_{a c t}$ is the activation overpotential expressed as follows:

$$
\eta_{\text {act }}=\phi_{\text {el }}-\phi_{\text {io }}-\eta_{\text {con }}
$$

where $\eta_{c o n}$ is the concentration overpotential expressed as follows:

$$
\eta_{\text {con }}=\frac{R T}{2 F} \ln \left(\frac{P_{\mathrm{H}_{2}, \text { bulk }}}{P_{\mathrm{H}_{2}}} \frac{P_{\mathrm{H}_{2} \mathrm{O}}}{P_{\mathrm{H}_{2} \mathrm{O}, \text { bulk }}}\right)
$$

The exchange current density $i_{0}$ in the Buttler-Volmer equation (eq.[8]) is assumed to have a linear dependency on the TPB density $l_{t p b}$ :

$$
i_{0}=i_{0, t p b} l_{t p b}
$$


where $i_{0, t p b}$ is the exchange current per unit TPB length. We use an empirical relationship for the $i_{0, t p b}(16,17)$ :

$\underline{\text { Boundary Conditions }}$

$$
i_{0, t p b}=31.4 P_{\mathrm{H}_{2}}^{-0.03} P_{\mathrm{H}_{2} \mathrm{O}}^{0.4} \exp \left(-\frac{18300}{T}\right)
$$

Boundary conditions used in the calculation are summarized in Table I. Gas compositions are constant on the anode surface to represent the supplied fuel composition. To determine the anode overpotential, the electric potential in the Ni phase at the anode surface and that in the YSZ phase at the anode-electrolyte interface are properly set.

TABLE I. Boundary Conditions.

\begin{tabular}{lll}
\hline \multicolumn{1}{c}{ Variables } & \multicolumn{1}{c}{ Surface $(\boldsymbol{x}=\mathbf{0})$} & Interface $(\boldsymbol{x}=\boldsymbol{L})$ \\
\hline $\mathrm{H}_{2}$ Partial Pressure & $P_{\mathrm{H}_{2}}(0)=P_{\mathrm{H}_{2}, \text { bulk }}$ & $\frac{d P_{\mathrm{H}_{2}}}{d x}(L)=0$ \\
$\mathrm{H}_{2} \mathrm{O}$ Partial Pressure & $P_{\mathrm{H}_{2} \mathrm{O}}(0)=P_{\mathrm{H}_{2} \mathrm{O}, \text { bulk }}$ & $\frac{d P_{\mathrm{H}_{2} \mathrm{O}}}{d x}(L)=0$ \\
Electric Potential in Ni & $\phi_{e l}(0)=\eta_{t}$ & $\frac{d \phi_{e l}}{d x}(L)=0$ \\
Electric Potential in YSZ & $\frac{d \phi_{i o}}{d x}(0)=0$ & $\phi_{i o}(L)=0$ \\
\hline
\end{tabular}

\section{Results and Discussion}

\section{Global Microstructural Parameters}

Prior to the numerical simulation, microstructural parameters, such as volume fraction, tortuosity factor, surface-to-volume ratio and TPB density, are quantified in the whole sample volume as "global" microstructural parameters. The results have been already reported in our previous works $(3,5,18)$. Therefore, we just summarize the sample sizes and voxel sizes in Table II, and quantified global microstructural parameters in Table III. Tortuosity factor is quantified by the random-walk-based diffusion simulation and TPB density by the volume expansion method.

TABLE II. Size and Resolutions of Anode Sample.

\begin{tabular}{lcccc}
\hline & Unit & $\mathbf{X}$ & $\mathbf{Y}$ & $\mathbf{Z}$ \\
\hline Size & {$[\mu \mathrm{m}]$} & 19.2 & 8.51 & 6.20 \\
Voxel Size & {$[\mathrm{nm}]$} & 26.6 & 26.6 & 62.0 \\
Resolution & & 720 & 320 & 100 \\
\hline
\end{tabular}

TABLE III. Quantified Global Microstructural Parameters.

\begin{tabular}{lccccc}
\hline & & Unit & Ni & YSZ & Pore \\
\hline Volume fraction & & {$[\%]$} & 25.3 & 25.1 & 49.6 \\
\hline \multirow{3}{*}{ Tortuosity factor } & $\mathrm{X}$ & {$[-]$} & 25.2 & 24.0 & 1.95 \\
& $\mathrm{Y}$ & {$[-]$} & 34.8 & 13.4 & 1.97 \\
& $\mathrm{Z}$ & {$[-]$} & 6.91 & 8.85 & 1.74 \\
\hline Surface-to-volume ratio & & {$\left[\mu \mathrm{m}^{2} / \mu \mathrm{m}^{3}\right]$} & 3.56 & 7.51 & 4.12 \\
\hline TPB density & & {$\left[\mu \mathrm{m} / \mu \mathrm{m}^{3}\right]$} & & 2.49 & \\
\hline
\end{tabular}




\section{$\underline{\text { Calculation Domain }}$}

Table IV summarizes the information of the grid systems used in this study. The control volume resolution is the number of image voxels contained in a control volume. Note that the characteristic scale of the porous structure is about $1 \mu \mathrm{m}$, considering the particle diameter of $\mathrm{Ni}$ and YSZ used in this study. The whole anode structure obtained by the FIB-SEM is mirror-symmetrically extended in the $Z$ direction for 5 times to realize the anode thickness of $31 \mu \mathrm{m}$.

TABLE IV. Size and Resolutions of Control Volume.

\begin{tabular}{rrrrrrrrrrr}
\hline & \multicolumn{3}{c}{ Control Volume Resolution } & \multicolumn{3}{c}{ Control Volume Size $[\boldsymbol{\mu m}]$} & \multicolumn{3}{c}{ Grid Number } \\
\cline { 2 - 10 } & $\mathrm{X}$ & $\mathrm{Y}$ & $\mathrm{Z}$ & $\mathrm{X}$ & $\mathrm{Y}$ & \multicolumn{1}{c}{$\mathrm{Z}$} & $\mathrm{X}$ & $\mathrm{Y}$ & \multicolumn{1}{c}{$\mathrm{Z}$} \\
\hline Grid 1 & 40 & 40 & 20 & 1.06 & 1.06 & 1.24 & 18 & 8 & $5 * 5$ \\
Grid 2 & 20 & 20 & 10 & 0.532 & 0.532 & 0.620 & 36 & 16 & $10 * 5$ \\
Grid 3 & 10 & 10 & 5 & 0.266 & 0.266 & 0.310 & 72 & 32 & $20 * 5$ \\
Grid 4 & 5 & 5 & 5 & 0.133 & 0.133 & 0.310 & 144 & 64 & $20 * 5$ \\
\hline
\end{tabular}

\section{Diffusion Simulation}

To confirm the validity of the proposed SGS models, we conduct the simple diffusion simulation prior to the anode overpotential analysis. We set the potential difference at the both edge sections perpendicular to the $Z$ direction and induce diffusion flux through the calculation domain. From the induced flux, we obtain the tortuosity factor of the whole calculation domain. If the obtained tortuosity factor is the same as the global microstructural parameter obtained by the random-walk simulation (Table III), we can judge that the amount of transport flux is appropriately evaluated and that the calculation domain reasonably represents the structural complexity of the porous anode.

If the size of the control volume is small enough, we can expect the calculation domain itself naturally represents the structural complexity. In contrast, if it is not, most of the structural information whose characteristic scale is smaller than the grid size is lost. Therefore, the proposed SGS models are required to be effective especially in the coarse grid case to correctly represent the structural complexity.

The calculated tortuosity factors in the Grid 1-4 with SGS1 and SGS2 are shown in Fig. 4. In these figures, horizontal dotted line represents the value obtained by the random-walk-based diffusion simulation. As expected, with the simple SGS model (SGS1), tortuosity factors are approaching to the dotted lines as the control volume size becomes smaller. However, the values are still underestimated because the information of structural inhomogeneity inside the grid is inevitably lost. Such tendency is prominent especially in the YSZ phase because of its small characteristic scale.

By correcting the transport flux with the SGS2, the trend in the figures is drastically changed; the amount of flux is modified to make the tortuosity factors approach to the global parameters. However, there is a difference in the trend among the phases. In the Ni and YSZ phases, tortuosity factors are reasonably evaluated even in the coarser grid. Especially in the Grid 2, the values are almost the same as the global parameters, which is a remarkable improvement by applying the SGS model. Even though there is a small overshoot in the Grid 3, the values tend to converge to the dotted lines. Therefore, it can 
be said that the SGS2 exerts its effect selectively in coarser grids, which is one of the most important aspect required for SGS model. In the pore phase, however, tortuosity factor is overestimated in the coarser grids, which indicates the flux modification is stronger than required. Although the different tendency may be attributed to the structural difference between the pore and the solid phases, the reason is not clear so far. It leaves a possibility to further improve the SGS model by considering the structure inside the control volume.
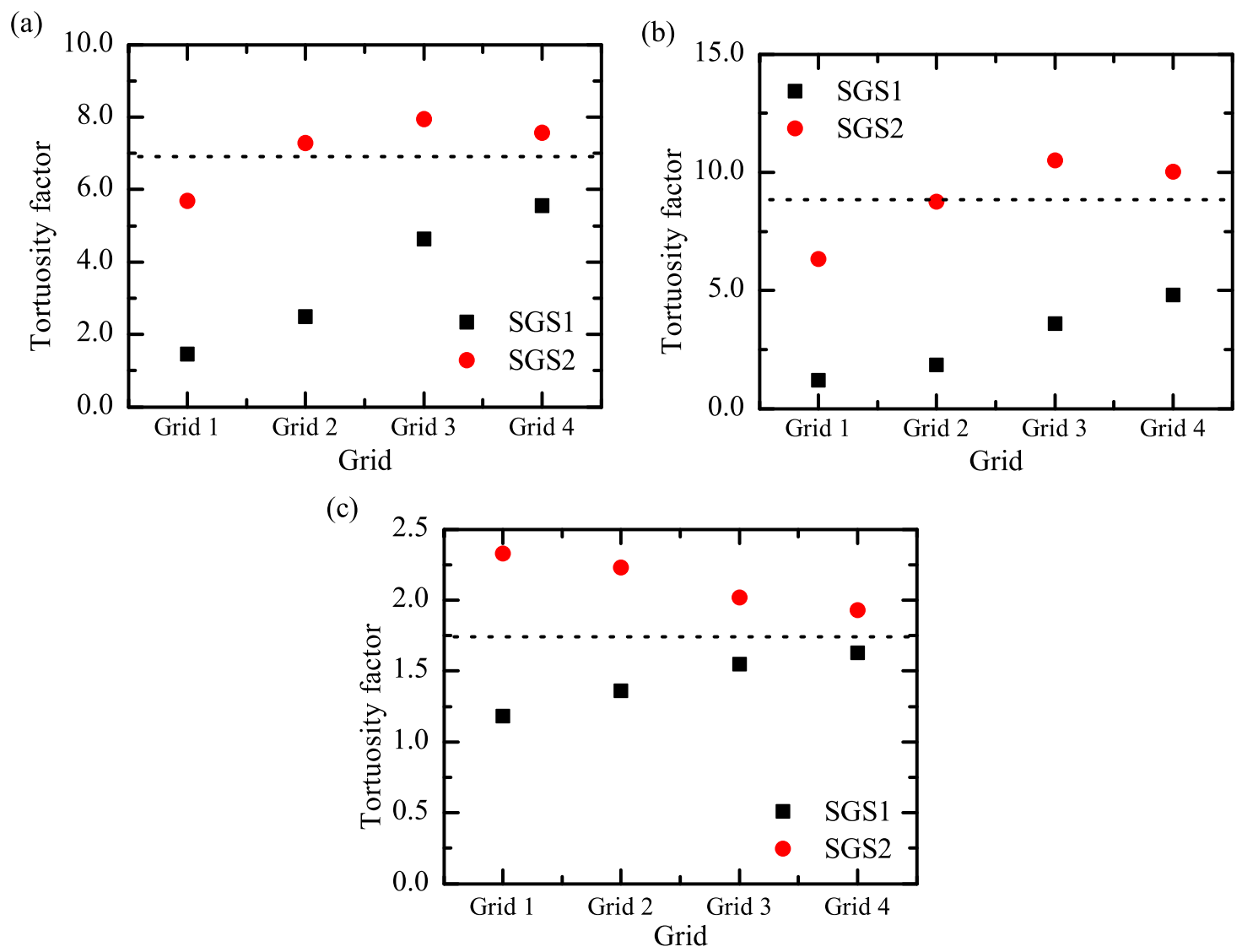

Figure 4. Tortuosity factors obtained by the diffusion calculation. (a) Ni phase (b) YSZ phase, (c) Pore phase.

\section{Anode Overpotential Prediction}

Numerical simulation to predict the anode polarization characteristics is conducted with the SGS models proposed in this study. Calculation parameters are summarized in Table V. Anode overpotential is set as a boundary condition and the average current density is obtained as a simulation result. Figure 5 shows the result obtained with each SGS model, and experimental result in our previous report [5] is also shown for comparison. With the simple SGS model (SGS1), polarization curves show strong dependency on the grid sizes (Fig. 5(a)). In contrast, they less depend on the grid size with the SGS2 (Fig. 5(b)). In spite of the variations of the evaluated tortuosity factors (Fig. 4), overpotential characteristics are less sensitive to the variation in the SGS2 case. This is consistent with the findings in our previous report (18). We conducted a sensitivity analysis to investigate the effect of microstructural parameters on the 
performance and found that the YSZ tortuosity factor had the largest influence. Its effect on the performance is non-linear and becomes prominent when the YSZ tortuosity factor is underestimated. This is the reason why the overpotential characteristics are almost the same in the SGS2 case.

Computation time required to obtain a convergent solution is shown in Fig. 6. Since it varies according to the applied SGS models and boundary conditions, the average values are shown with error bars. In each calculation, we use a single core of Core 2 Duo E8600 processor. The time required in the Grid 2 is more than 30 times less than that in the Grid 4. It clearly shows the effectiveness of the proposed SGS model. If coarser grid can be used in the simulation without losing the simulation accuracy, it can be applied to an electrode simulation in an SOFC macro-scale analysis. We believe the proposed SGS model has a potential to act as a bridge builder between micro-scale analysis and macro-scale analysis.

TABLE V. Calculation Parameters.

\begin{tabular}{lccc}
\hline & Unit & Symbol & Value \\
\hline Total pressure & {$[\mathrm{Pa}]$} & $P_{t}$ & $1.013 \times 10^{5}$ \\
Temperature & {$[\mathrm{K}]$} & $T$ & 1273 \\
$\mathrm{H}_{2}$ partial pressure & {$[\mathrm{Pa}]$} & $P_{\mathrm{H} 2, \text { bulk }}$ & $0.97 \times P_{t}$ \\
$\mathrm{H}_{2} \mathrm{O}$ partial pressure & {$[\mathrm{Pa}]$} & $P_{\mathrm{H} 2 \mathrm{O}, \text { bulk }}$ & $0.03 \times P_{t}$ \\
Anode thickness & {$[\mu \mathrm{m}]$} & $L$ & 31 \\
\hline
\end{tabular}

(a)

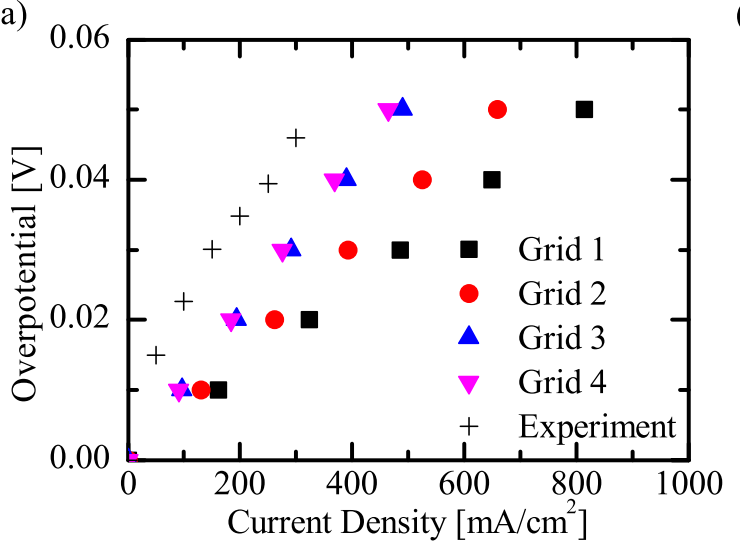

(b)

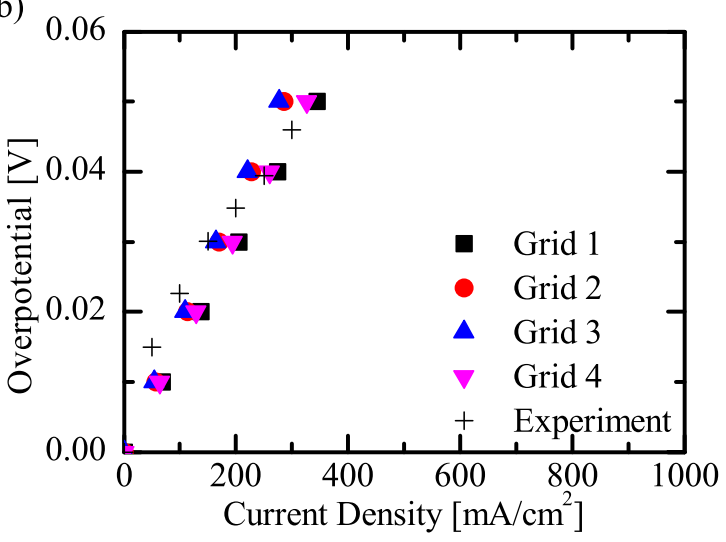

Figure 5. Predicted anode overpotentials with SGS models. (a)SGS1. (b)SGS2.

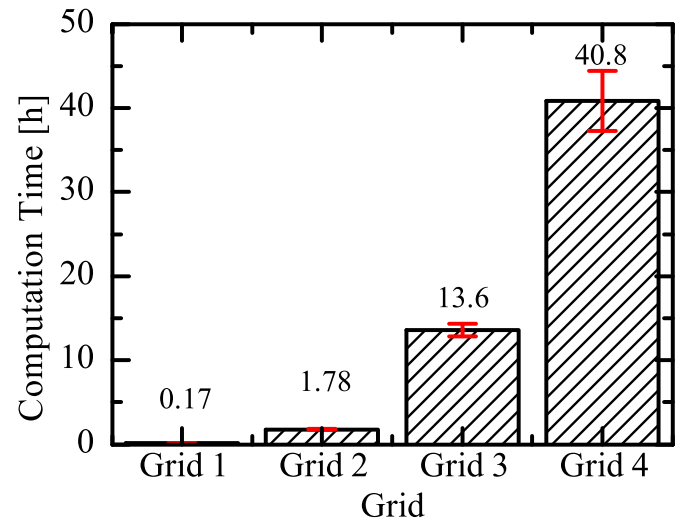

Figure 6. Computation time required to obtain convergent solution. 


\section{Conclusions}

Three-dimensional simulation to predict the SOFC anode polarization is performed with a structure obtained by the FIB-SEM. A sub-grid scale model (SGS) is newly developed and effectively used to evaluate the transport flux in the porous structure. By considering the phase connectivity between the neighboring grids, structural complexity of the porous anode is reasonably taken into account. As a result, transport flux through the porous anode is properly evaluated, and anode polarization characteristics are reasonably predicted even when coarser grid is used. The proposed SGS model has a potential to combine electrode simulation with much larger scale analysis, such as SOFC macro-scale analysis with reasonable calculation load.

\section{Acknowledgments}

This work was supported by the New Energy and Industrial Technology Development Organization (NEDO) under the Development of System and Elemental Technology on Solid Oxide Fuel Cell (SOFC) Project.

\section{References}

1. J.R. Wilson et al., Nature Material, 5(7), 541 (2006).

2. P.R. Shearing et al., Chem. Eng. Sci., 64(17), 3928 (2009).

3. H. Iwai et al., J. Power Sources, 195, 955 (2010).

4. D. Gostovic et al., Electrochem. Solid-State Lett., 10(12), B214 (2007).

5. M. Kishimoto et al., "Quantitative Evaluation of SOFC Porous Anode Microstructure Based on FIB-SEM Technique and Prediction of Anode Overpotentials", J. Power Sources, (2011) Accepted.

6. N. Shikazono et al., J. Electrochem. Soc., 157(5), B665 (2010).

7. P.R. Shearing et al., J. Power Sources, 195, 4804 (2010).

8. D. Simwonis et al., Solid State Ionics, 132, 241 (2000).

9. J.-H. Lee et al., Solid State Ionics, 148, 15 (2002).

10. U. Anselmi-Tamburini et al., Solid State Ionics, 110, 35 (1998).

11. N.F. Bessette II et al., J. Electrochem. Soc., 142(11), 3792 (1995).

12. E.A. Mason et al., J. Chem. Physics, 46(8), 3199 (1967).

13. D. Aronšt et al., The Chem. Eng. J., 57, 91 (1995).

14. E.N. Fuller et al., Industrial and Eng. Chemistry Research, 58(5) 19 (1966).

15. R. H. Perry, Perry's Chemical Engineers' Handbook $7^{\text {th }}$ Edition, McGraw-Hill, New York (1981).

16. B. De Boer, Ph.D. thesis, University of Twente, The Netherland (1998).

17. Y. Suzue et al., J. Power Sources, 184, 52 (2008).

18. M. Kishimoto et al., The $14^{\text {th }}$ Int. Heat Transfer Conference, IHTC-22495 (2010). 